\title{
Waist-to-Hip Ratio, Cardiovascular Outcomes, and Death in Peritoneal Dialysis Patients
}

\author{
Winnie S. Su, ${ }^{1}$ Catherine M. Clase, ${ }^{1,2}$ K. Scott Brimble, ${ }^{1}$ Peter J. Margetts, ${ }^{1}$ \\ Trevor J. Wilkieson, ${ }^{1}$ and Azim S. Gangji ${ }^{1,2}$ \\ ${ }^{1}$ Division of Nephrology, McMaster University, St. Joseph's Healthcare, Hamilton, ON, Canada L8N 4A6 \\ ${ }^{2}$ Department of Clinical Epidemiology and Biostatistics, McMaster University, Hamilton, ON, Canada L8N 4A6
}

Correspondence should be addressed to Azim S. Gangji, gangji@mcmaster.ca

Received 9 February 2010; Revised 16 April 2010; Accepted 25 May 2010

Academic Editor: Ramesh Khanna

Copyright (C) 2010 Winnie S. Su et al. This is an open access article distributed under the Creative Commons Attribution License, which permits unrestricted use, distribution, and reproduction in any medium, provided the original work is properly cited.

\begin{abstract}
Objectives. The primary objective of this study was to determine the relationship between waist-to-hip ratio (WHR), cardiovascular (CV) events, and mortality in peritoneal dialysis (PD) patients. A secondary objective was to investigate the association between abdominal obesity and systemic inflammatory markers. Methods. This is a prospective study of 22 prevalent PD patients. WHR was measured at baseline. C-reactive protein (CRP), tumour necrosis factor- $\alpha$ (TNF- $\alpha$ ), and interleukin-6 (IL-6) were measured. Main outcomes were first CV event and death from all causes. Survival analysis was used to examine the relationship between anthropomorphic measures and clinical outcomes. Results. Mean follow-up period was 3.1 years. In Kaplan-Meier analysis, survival was lower in those with higher WHR $(P=.002)$. In Cox regression, WHR independently predicted mortality and first CV event after adjustment for known ischemic heart disease (hazard ratio [HR] 1.17, confidence interval [CI] 1.05-1.30 for death; HR 1.13, CI 1.01-1.26 for CV event). WHR correlated with serum TNF- $\alpha(r=0.45 ; P=.05)$. Conclusion. The results of this study suggest WHR may be a risk factor for increased CV events and mortality in PD patients. Abdominal obesity is also associated with inflammatory markers. Larger studies are warranted to confirm these findings.
\end{abstract}

\section{Introduction}

Cardiovascular $(\mathrm{CV})$ disease is the leading cause of death in dialysis patients [1]. Obesity increases the risk of $\mathrm{CV}$ disease in the general population [2]. In particular, abdominal obesity as measured by waist-to-hip ratio (WHR) has emerged as a better predictor of CV risk than body mass index (BMI) [3]. In several large epidemiologic studies, WHR was an independent predictor of coronary artery disease and death [3-5].

Studies on abdominal obesity in patients with chronic kidney disease are limited. In one study of patients with mild to moderate chronic kidney disease (CKD), increased WHR predicted fatal and nonfatal coronary artery disease events [6]. The first study of WHR in hemodialysis (HD) patients was recently published in [7]. Higher WHR and waist circumference (WC) predicted CV and all-cause death after adjusting for BMI whereas higher BMI was protective [7]. To our knowledge, there are no studies in peritoneal dialysis (PD) patients that have assessed the relationship between WHR and clinically important adverse events.

One of the postulated mechanisms for abdominal obesity's detrimental effect is its relationship with inflammatory markers, and inflammation is associated with increased CV risk in chronic kidney disease $[8,9]$. Excess abdominal fat has been shown to be associated with increased C-reactive protein (CRP), tumor necrosis factor-alpha (TNF- $\alpha$ ), and interleukin-6 (IL-6) in general populations $[10,11]$, but studies in PD patients are scarce [12].

The primary objective of this study was to investigate the relationship between central obesity as measured by WHR, and CV events and death in PD patients. A secondary objective was to determine if there was an association between anthropomorphic measures and systemic inflammatory markers. 


\section{Methods}

This is a prospective study of 22 prevalent, stable, and consenting PD patients who were recruited from one of the affiliate teaching hospitals associated with McMaster University, (St. Joseph's Healthcare), Hamilton, Canada. Study protocol was approved by the St. Joseph's Healthcare Research Ethics Board. Patients were included in the study if they were 18 years of age or older and on PD for at least 3 months. Exclusion criteria included a history of peritonitis in the previous month and inability to provide informed consent.

Patient charts were reviewed to collect demographic information, a list of comorbidities, medications, and dialysis prescription. Transport property of the peritoneal membrane was determined by the fast peritoneal equilibration test [13], and transport status was established based on the four-hour dialysate/plasma $(\mathrm{d} / \mathrm{p})$ creatinine. Residual renal function was calculated as the mean of 24-hour urea and creatinine clearances.

On the day of testing, two hours into the dwell for the peritoneal equilibration test, serum IL-6, TNF- $\alpha$, and CRP (Cardiophase* hs CRP Dade Behring Inc. Newark, DE) levels were drawn. IL- 6 and TNF- $\alpha$ were measured by ELISA (R\&D Systems, Minneapolis MN).

Weight and height were measured without shoes. Measurements and patient assessment were completed after patients had drained their overnight dialysate dwell. Waist circumference (WC) was measured around the narrowest point between the coastal margin and the iliac crest. Hip circumference was measured at the level of the widest diameter around the gluteal region. Waist-to-hip ratio is waist circumference divided by hip circumference. BMI is weight divided by (height) ${ }^{2}$.

Patients were recruited between March and September of 2005. They were followed until December 2008 or death. The main outcomes were first $\mathrm{CV}$ event (myocardial infarction [MI], stroke, amputation, cardiac revascularization, or CV death); only one event was included for the analysis and death from all causes. MI was diagnosed when two out of three of the following criteria were met: typical symptoms, elevation in cardiac enzyme (troponin $\mathrm{T}>0.1 \mathrm{mcg} / \mathrm{L}$ ), or diagnostic changes on the electrocardiogram. Stroke was defined as new neurologic deficit thought to be vascular in origin and lasting more than 24 hours. CV death included death caused by MI, stroke, complications of peripheral arterial disease, and all sudden death.

\section{Statistics}

All analyses were performed with the statistical package, SPSS (version 17; SPSS Inc. Chicago, IL). Subjects were divided in to two groups according to the median value for their gender for each anthropomorphic measure. MannWhitney $U$ test was used to compare continuous baseline variables between groups. Fisher exact test was used to compare categorical CV risk factors between groups. Bivariate correlations are reported using the Spearman rank correlation coefficient. Two-sided probability $(P)$ values were
TABLe 1: Baseline characteristics of the 22 peritoneal dialysis patients enrolled.

\begin{tabular}{|c|c|}
\hline Variable & $\begin{array}{c}\text { PD Patients } N=22 \\
n(\%) \text { or Mean } \\
\text { (Standard Deviation) }\end{array}$ \\
\hline Age (years) & $61(\mathrm{SD} 15.6)$ \\
\hline Male & $13(59 \%)$ \\
\hline Caucasian & $21(95 \%)$ \\
\hline Time on dialysis (years) & $2.1(\mathrm{SD} 2.8)$ \\
\hline Diabetes Mellitus & $8(37 \%)$ \\
\hline Ischemic heart disease & $5(23 \%)$ \\
\hline Peripheral arterial disease & $3(14 \%)$ \\
\hline Hypertension & $18(82 \%)$ \\
\hline Any history of smoking & $11(50 \%)$ \\
\hline Waist Circumference $(\mathrm{cm})$ & 101 (SD 11.5) \\
\hline Men & 104 (SD 12.6) \\
\hline Women & $97.1(\mathrm{SD} 8.85)$ \\
\hline Waist-to-Hip Ratio & 0.98 (SD 0.09) \\
\hline Men & $1.01(\mathrm{SD} 0.08)$ \\
\hline Women & $0.94(\mathrm{SD} 0.09)$ \\
\hline $\operatorname{BMI}\left(\mathrm{kg} / \mathrm{m}^{2}\right)$ & 27.7 (SD 4.94) \\
\hline Men & $28.2(\mathrm{SD} 5.14)$ \\
\hline Women & $27.1(\mathrm{SD} 4.87)$ \\
\hline
\end{tabular}

calculated with statistical significance set at alpha $\leq .05$. Univariate comparisons of survival between those with high or low WHR were performed with Kaplan-Meier analysis and log-rank tests. The relationships between WHR and clinical outcomes were also investigated using Cox regression. Logistic regression was used if assumption of proportionality was violated. Baseline variables found to be significantly different between high and low WHR groups were entered into a Cox regression model as covariate. However, the number of variables entering the model was limited by the number of events to ensure model stability [14].

\section{Results}

All 43 eligible patients at our centre were approached, and 22 patients consented. The majority of the patients declined due to time commitment and need for followup. All enrolled patients were followed to the end of the study.

Baseline characteristics of the 22 patients are summarized in Table 1 . Mean age \pm standard deviation was $61 \pm 16$ years; 59\% were men, and 95\% were Caucasian. At baseline, 37\% had diabetes (DM), and 23\% had known ischemic heart disease (IHD). Median WHR was 1.01 for men and 0.95 for women.

After a mean follow-up period of $3.1 \pm 0.81$ years, 9 patients $(41 \%)$ had at least one CV event. There were 9 deaths ( $41 \%$ mortality), and 5 out of the 9 deaths were related to CV causes ( 3 cardiac events, 1 stroke, and 1 ruptured abdominal aortic aneurysm). 4 out $5 \mathrm{CV}$ deaths occurred in those with WHR above the gender-specific median. The other 4 deaths 


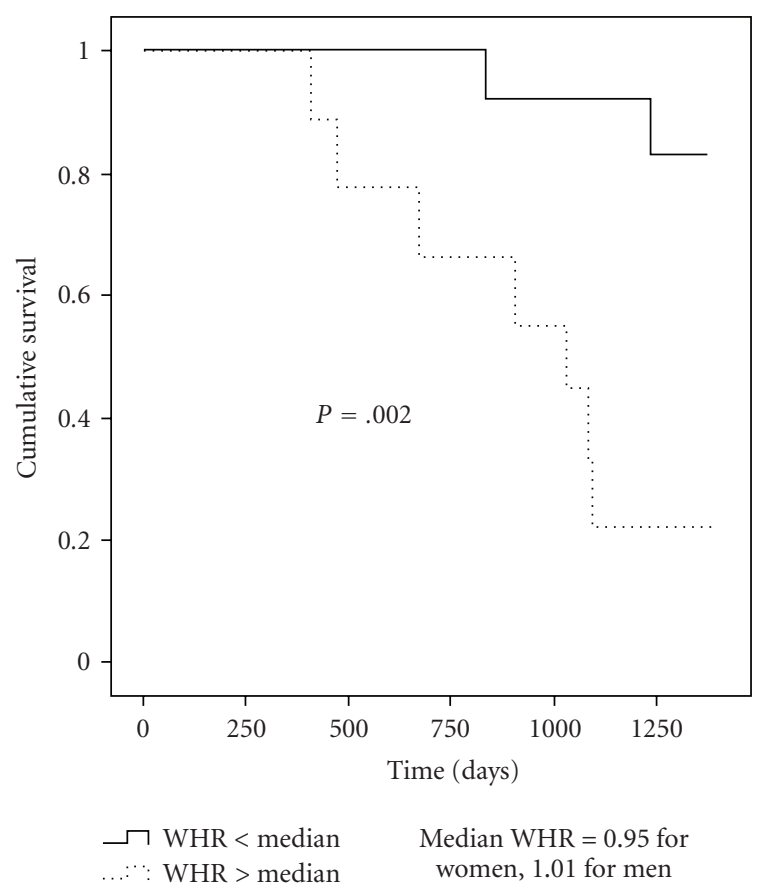

FIgURE 1: Kaplan-Meier survival curves for patients above and below the gender-specific median for WHR.

were due to infection or malignancy ( 2 with high WHR and 2 with low WHR). There were 9 first CV events; 6 occurred in those with high WHR (5 MIs and 1 stroke), and 3 occurred in those with low WHR (all MIs).

$\mathrm{CV}$ risk factors and other baseline characteristics with known prognostic significance in PD patients were compared between the two groups of patients with WHR above and below the gender-specific medians. There were no significant differences in median age (61 and 62; $P=.74$ ), male gender (67\% and 54\%; $P=.67$ ), the presence of hypertension (89\% and $77 \% ; P=.62)$, DM $(56 \%$ and $23 \% ; P=.19)$, any smoking history $(67 \%$ and $54 \% ; P=.67)$, known IHD ( $44 \%$ and $15 \% ; P=.18)$, median albumin $(38$ and $36 \mathrm{~g} / \mathrm{L}$; $P=.90$ ), peritoneal transport characteristics (high or high average $100 \%$ and $77 \%, P=.24$ ), residual renal function (4.36 and $4.65 \mathrm{~mL} / \mathrm{min} ; P=.79)$, and transfer to HD (22 and $15 \% ; P=1.0)$. There were also no differences in $\mathrm{PD}$ modality (automated versus continuous ambulatory PD), $(P=.41)$ use of icodextrin, $(P=.36)$, or peritonitis rates $(P=1.0)$.

Kaplan-Meier survival analysis showed a lower cumulative survival $(P=.002)$ in those with higher WHR (Figure 1). There were also increased overall CV events $(P=.03)$ and CV deaths $(P=.01)$ in the group with higher WHR. KaplanMeier survival analysis was not performed for WC and BMI due to violation of the assumption of proportionality.

Using Cox regression, the hazard ratio (HR) for death with every 0.01 change in WHR was 1.15 (confidence interval [CI] 1.05-1.24), and for first CV event, the HR was 1.13 (CI 1.03-1.25). None of the measured baseline characteristics were significantly different between high and low WHR groups. However, because of a trend towards increased IHD
TABLE 2: Correlations between systemic inflammatory markers and anthropomorphic measures.

\begin{tabular}{lccc}
\hline & $\begin{array}{c}\text { Waist-to-Hip } \\
\text { ratio } \\
r(P)\end{array}$ & $\begin{array}{c}\text { Waist } \\
\text { circumference } \\
r(P)\end{array}$ & $\begin{array}{c}\text { Body mass index } \\
r(P)\end{array}$ \\
\hline $\begin{array}{l}\text { Inflammatory } \\
\text { markers }\end{array}$ & & & \\
CRP & $\mathrm{NS}$ & $0.45(P=.04)$ & $\mathrm{NS}$ \\
TNF- $\alpha$ & $0.45(P=.05)$ & $0.58(P=.007)$ & NS \\
IL-6 & $\mathrm{NS}$ & $\mathrm{NS}$ & $\mathrm{NS}$ \\
\hline
\end{tabular}

$r$ : correlation coefficient, CRP: C-reactive protein, TNF- $\alpha$ : tumor necrosis factor- $\alpha$, IL-6: interleukin-6, and NS: nonsignificant.

and DM in the high WHR group and the known association with poor outcomes in dialysis patients, IHD and DM were entered as covariates in Cox regression models. After adjusting for IHD, HR for death was 1.17 (CI 1.05-1.30), and HR for first CV event was 1.13 (CI 1.01-1.26) in patients with high WHR. After adjusting for DM, high WHR patients had a HR for death equal to 1.16 (CI 1.05-1.28), and HR for first CV event was 1.12 (CI 1.01-1.24).

Using logistic regression, neither WC nor BMI predicted death (odds ratio [OR $1.06 \mathrm{CI} 0.97-1.16$ for WC and OR 1.03 CI $0.86-1.22$ for BMI, resp.) or CV events (OR $1.07 \mathrm{CI}$ 0.98-1.17 for WC and OR 1.06 CI 0.89-1.26 for BMI, resp.).

WHR correlated with TNF- $\alpha$ but not with CRP or IL6 (Table 2). WC correlated with TNF- $\alpha$ and CRP. BMI did not correlate with measured inflammatory markers. No significant correlations were found between serum inflammatory markers and DM, known IHD, cholesterol profile, or peritoneal membrane transport characteristics.

\section{Discussion}

The main finding of this study is that increased WHR is associated with increased CV events and all-cause mortality in PD patients. To our knowledge, this is the first report of such a relationship in PD patients. These results are consistent with what has previously been identified in the general population in [4], in patients with CKD in [6], and those on HD in [7]. Our data is also consistent with results showing that abdominal obesity is a stronger adverse prognosticator than BMI and that WHR may be a better measurement of abdominal adiposity than $\mathrm{WC}[3,6]$. In the INTERHEART study, BMI showed the weakest association with myocardial infarction whereas waist circumference was intermediate between WHR and BMI [3]. In HD patients, WC was a significant predictor of CV and all-cause mortality only after adjustment for BMI whereas WHR was predictive of mortality both before and after adjustment for BMI [7]. Although there was a high mortality rate of $41 \%$ during a mean follow-up period of 3.1 years in our study, this result is in keeping with the known morality rate of the Canadian dialysis population $[15,16]$.

In the general population, higher BMI predicts mortality, but the relationship appears to differ for patients on dialysis [17]. Studies in HD patients have shown paradoxically 
increased survival with higher BMI [17], but results in PD patients are mixed [18]. In our study, there was no association between increased BMI and clinical outcomes. This neutral relationship was also found in several other studies in PD patients $[18,19]$. Some have postulated that protective effects of elevated BMI may be neutralized by increased PD-related infection, underdialysis, or more rapid loss of residual renal function [20]. The conflicting findings may also be in part related to the inability of BMI to differentiate between fat, muscle tissue, and water. BMI reflects a combination of multiple factors including subcutaneous fat, visceral fat, muscle mass, and nutritional and hydration status [21]; these factors may have mixed and competing effects on survival [22]. Therefore, BMI does not seem to be a reliable prognostic factor in PD patients. On the other hand, WHR is primarily a measure of visceral fat which is thought to be particularly detrimental due to its proatherogenic and proinflammatory properties [10].

In this study, increased WHR is associated with higher levels of TNF- $\alpha$, and increased WC is associated with both higher levels of TNF- $\alpha$ and CRP. These correlations are consistent with the existing hypothesis that visceral fat has important inflammatory properties. Studies in the general population have shown higher circulating levels of CRP, TNF- $\alpha$, and IL- 6 in those with increased abdominal fat when compared with BMI-matched controls [10,23]. In one small study of PD patients, WHR was significantly associated with IL-6 but not with CRP, or TNF- $\alpha$ [12]. However, factors other than abdominal obesity such as chronic infections, volume overload, accumulation of advanced glycation end-products, and peritoneal membrane inflammation can potentially contribute to systemic inflammation in PD [24]. These factors may explain differences in results between studies and the lack of association between WHR and CRP or IL-6 in our study.

When using gender-specific cutoffs for abdominal obesity defined by WC [25], the prevalence of abdominal obesity in this study (64\%) is higher compared with that previously reported in HD patients (39\%) in [7]. The mean WHR $(0.98$ \pm 0.09 ) in our cohort was also higher than that reported in HD patients $(0.93 \pm 0.10)$ [7]. PD patients may be at higher risk of developing abdominal obesity compared with HD patients because of the use of glucose-based dialysis solutions. Glucose loading in $\mathrm{PD}$ has been postulated to contribute to hyperinsulinemia and insulin resistance $[26,27]$; these factors are in turn highly associated with abdominal obesity $[11,28]$. In one study, patients developed a 23\% increase in intra-abdominal fat area after initiating PD, despite a lack of significant increase in weight or proportion of total fat mass [29]. Abdominal obesity may therefore be a particularly important consideration in PD patients, and this highlights the importance of clinical evaluation of glucose sparing PD prescriptions [30]. Randomized trials of glucose-sparing strategies using new dialysate solutions are underway.

The strength of this study is the prospective design, longitudinal followup, and completeness of followup (100\%). The main limitation of this study is the small sample size, which precludes extensive multivariate analysis. After adjusting for
IHD and DM, WHR remained an independent risk factor for mortality and first CV event. Although there was no difference in the presence of other major CV risk factors (age, gender, diabetes, hypertension, and smoking history) or known adverse prognostic variables in PD patients (serum albumin, peritoneal transport characteristics, and residual renal function) between patients with high or low WHR, it is possible that significant differences were not detected due to the small sample size. It may be that WHR is associated with a number of other risk factors however adjustment for additional potential confounding factors in the Cox regression model was also not possible with the small sample size [14]. This study should therefore be regarded as hypothesis-generating, and the preliminary data presented here provides justification for larger studies in the future.

In conclusion, this small study suggests increased WHR may be a risk factor for $\mathrm{CV}$ events and mortality in $\mathrm{PD}$ patients. WHR is a simple measurement that can be incorporated in clinical practice, and larger studies with multivariate analysis are warranted to confirm the relationship between WHR and clinical outcomes in PD patients.

\section{Conflict of Interest}

Drs. W. Su, C. Clase, K. Brimble, P. Margetts, A. Gangji, and Mr. T. Wilkieson do not have any conflicts of interest.

\section{References}

[1] R. N. Foley, P. S. Parfrey, and M. J. Sarnak, "Clinical epidemiology of cardiovascular disease in chronic renal disease," American Journal of Kidney Diseases, vol. 32, no. 5, supplement 3, pp. S112-S119, 1998.

[2] J. E. Manson, G. A. Colditz, M. J. Stampfer et al., "A prospective study of obesity and risk of coronary heart disease in women," New England Journal of Medicine, vol. 322, no. 13, pp. 882-889, 1990.

[3] S. Yusuf, S. Hawken, S. Ônpuu et al., "Obesity and the risk of myocardial infarction in 27000 participants from 52 countries: a case-control study," Lancet, vol. 366, no. 9497, pp. 1640-1649, 2005.

[4] T. Pischon, H. Boeing, K. Hoffmann et al., "General and abdominal adiposity and risk of death in Europe," New England Journal of Medicine, vol. 359, no. 20, pp. 2105-2120, 2008.

[5] C. Zhang, K. M. Rexrode, R. M. van Dam, T. Y. Li, and F. B. $\mathrm{Hu}$, "Abdominal obesity and the risk of all-cause, cardiovascular, and cancer mortality: sixteen years of followup in US women," Circulation, vol. 117, no. 13, pp. 1658-1667, 2008.

[6] E. F. Elsayed, H. Tighiouart, D. E. Weiner et al., "Waist-to-hip ratio and body mass index as risk factors for cardiovascular events in CKD," American Journal of Kidney Diseases, vol. 52, no. 1, pp. 49-57, 2008.

[7] M. Postorino, C. Marino, G. Tripepi, and C. Zoccali, "Abdominal obesity and all-cause and cardiovascular mortality in end-stage renal disease," Journal of the American College of Cardiology, vol. 53, no. 15, pp. 1265-1272, 2009. 
[8] P. W. B. Nanayakkara, C. Y. Le Poole, D. Fouque et al., "Plasma adiponectin concentration has an inverse and a non linear association with estimated glomerular filtration rate in patients with K/DOQI 3-5 chronic kidney disease," Clinical Nephrology, vol. 72, no. 1, pp. 21-30, 2009.

[9] S.-H. Park, J. J. Carrero, B. Lindholm, and P. Stenvinkel, "Adiponectin in chronic kidney disease has an opposite impact on protein-energy wasting and cardiovascular risk: two sides of the same coin," Clinical Nephrology, vol. 72, no. 2, pp. 8796, 2009.

[10] A. H. Berg and P. E. Scherer, "Adipose tissue, inflammation, and cardiovascular disease," Circulation Research, vol. 96, no. 9, pp. 939-949, 2005.

[11] J.-P. Després and I. Lemieux, "Abdominal obesity and metabolic syndrome," Nature, vol. 444, no. 7121, pp. 881-887, 2006.

[12] T. Stompór, W. Sulowicz, A. Dembińska-Kieć, K. Janda, K. Wójcik, and A. Zdzienicka, "An association between body mass index and markers of inflammation: is obesity the proinflammatory state in patients on peritoneal dialysis?" Peritoneal Dialysis International, vol. 23, no. 1, pp. 79-83, 2003.

[13] Z. J. Twardowski, "PET-a simpler approach for determining prescriptions for adequate dialysis therapy," Advances in Peritoneal Dialysis, vol. 6, pp. 186-191, 1990.

[14] B. G. Tabachnick and F. S. Fidell, Using Multivariate Statistics, Allyn and Bacon, Boston, Mass, USA, 5th edition, 2006.

[15] Canadian Institute for Health Information, "Canadian Organ Replacement Register: five year survival of ESRD patients on dialysis, with and without diabetes, by age, 1997-2004," Ottawa, Ontario, Canada, June 2010, http://secure.cihi.ca/ cihiweb/en/corr_quickstats_200907_fig1_e.html.

[16] M. Tonelli, B. Hemmelgarn, B. Culleton et al., "Mortality of Canadians treated by peritoneal dialysis in remote locations," Kidney International, vol. 72, no. 8, pp. 1023-1028, 2007.

[17] K. Kalantar-Zadeh, J. D. Kopple, R. D. Kilpatrick et al., "Association of morbid obesity and weight change over time with cardiovascular survival in hemodialysis population," American Journal of Kidney Diseases, vol. 46, no. 3, pp. 489500, 2005.

[18] T. Pliakogiannis, L. Trpeski, H. Taskapan et al., "Reverse epidemiology in peritoneal dialysis patients: the Canadian experience and review of the literature," International Urology and Nephrology, vol. 39, no. 1, pp. 281-288, 2007.

[19] A. G. Stack, B. V. R. Murthy, and D. A. Molony, "Survival differences between peritoneal dialysis and hemodialysis among "large" ESRD patients in the United States," Kidney International, vol. 65, no. 6, pp. 2398-2408, 2004.

[20] D. W. Johnson, "What is the optimal fat mass in peritoneal dialysis patients?" Peritoneal Dialysis International, vol. 27, no. 2, pp. S250-S254, 2007.

[21] A. H. Tzamaloukas, G. H. Murata, B. Piraino et al., "Sources of variation in estimates of lean body mass by creatinine kinetics and by methods based on body water or body mass index in patients on continuous peritoneal dialysis," Journal of Renal Nutrition, vol. 20, no. 2, pp. 91-100, 2010.

[22] B. C. H. Kwan and S. Beddhu, "A story half untold: adiposity, adipokines and outcomes in dialysis population," Seminars in Dialysis, vol. 20, no. 6, pp. 493-497, 2007.

[23] J.-P. Després, "Cardiovascular disease under the influence of excess visceral fat," Critical Pathways in Cardiology, vol. 6, no. 2, pp. 51-59, 2007.
[24] R. Pecoits-Filho, P. Stenvinkel, A. Yee-Moon Wang, O. Heimbürger, and B. Lindholm, "Chronic inflammation in peritoneal dialysis: the search for the holy grail?" Peritoneal Dialysis International, vol. 24, no. 4, pp. 327-339, 2004.

[25] National Cholesterol Education Program, "Third Report of the National Cholesterol Education Program (NCEP) Expert Panel on Detection, Evaluation, and Treatment of High Blood Cholesterol in Adults (Adult Treatment Panel III) final report," Circulation, vol. 106, no. 25, pp. 3143-3421, 2002.

[26] G. Amici, M. Orrasch, G. Da Rin, and C. Bocci, "Hyperinsulinism reduction associated with icodextrin treatment in continuous ambulatory peritoneal dialysis patients," Advances in Peritoneal Dialysis, vol. 17, pp. 80-83, 2001.

[27] M. Canbakan and G. M. Şahin, "Icodextrine and insulin resistance in continuous ambulatory peritoneal dialysis patients," Renal Failure, vol. 29, no. 3, pp. 289-293, 2007.

[28] R. N. Bergman, S. P. Kim, I. R. Hsu et al., "Abdominal obesity: role in the pathophysiology of metabolic disease and cardiovascular risk," American Journal of Medicine, vol. 120, no. 2, supplement 1, pp. S3-S8, 2007.

[29] A. Fernström, B. Hylander, Å. Moritz, H. Jacobsson, and S. Rössner, "Increase of intra-abdominal fat in patients treated with continuous ambulatory peritoneal dialysis," Peritoneal Dialysis International, vol. 18, no. 2, pp. 166-171, 1998.

[30] C. Holmes and S. Mujais, "Glucose sparing in peritoneal dialysis: implications and metrics," Kidney International. Supplement, no. 103, pp. S104-109, 2006. 


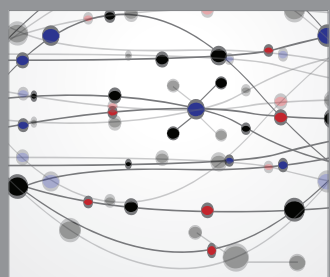

The Scientific World Journal
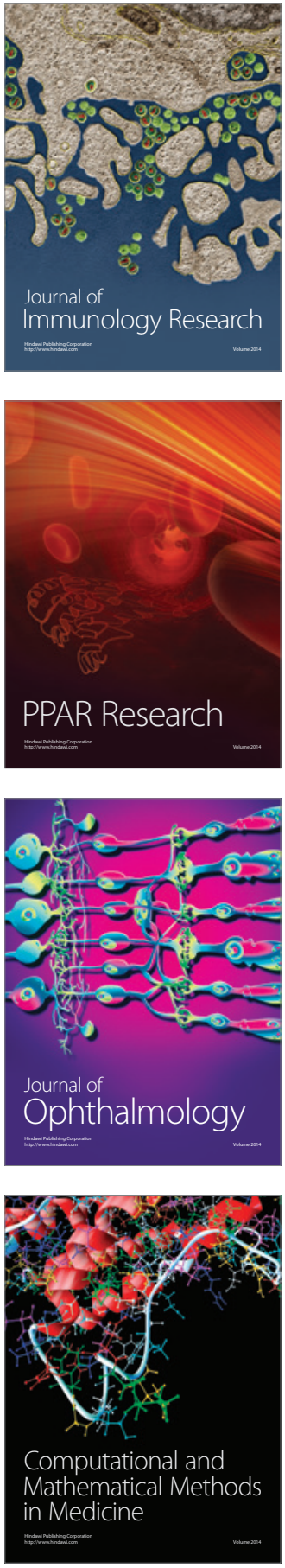

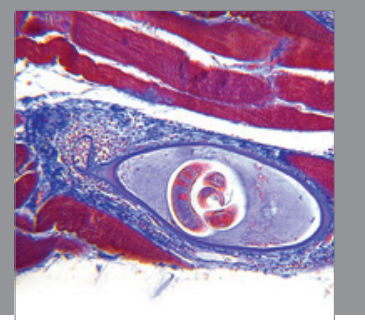

Gastroenterology

Research and Practice
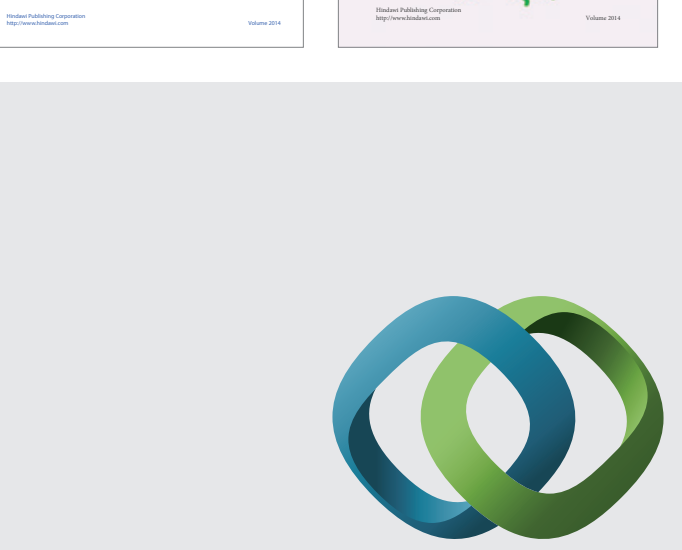

\section{Hindawi}

Submit your manuscripts at

http://www.hindawi.com
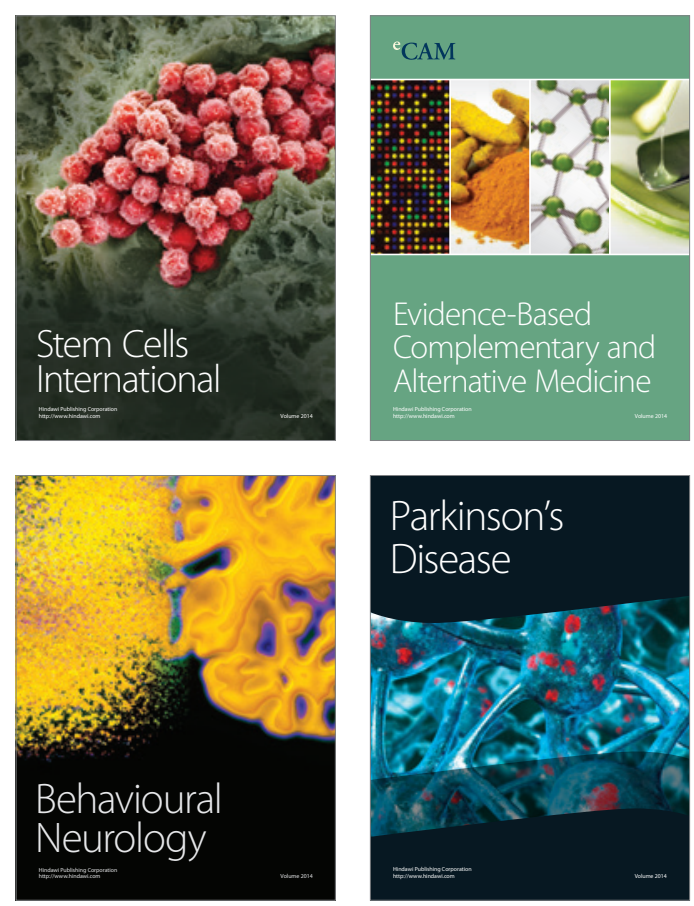

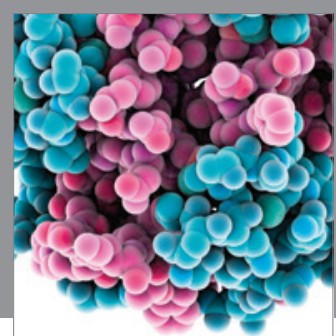

Journal of
Diabetes Research

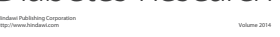

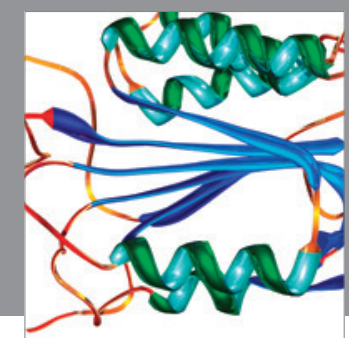

Disease Markers
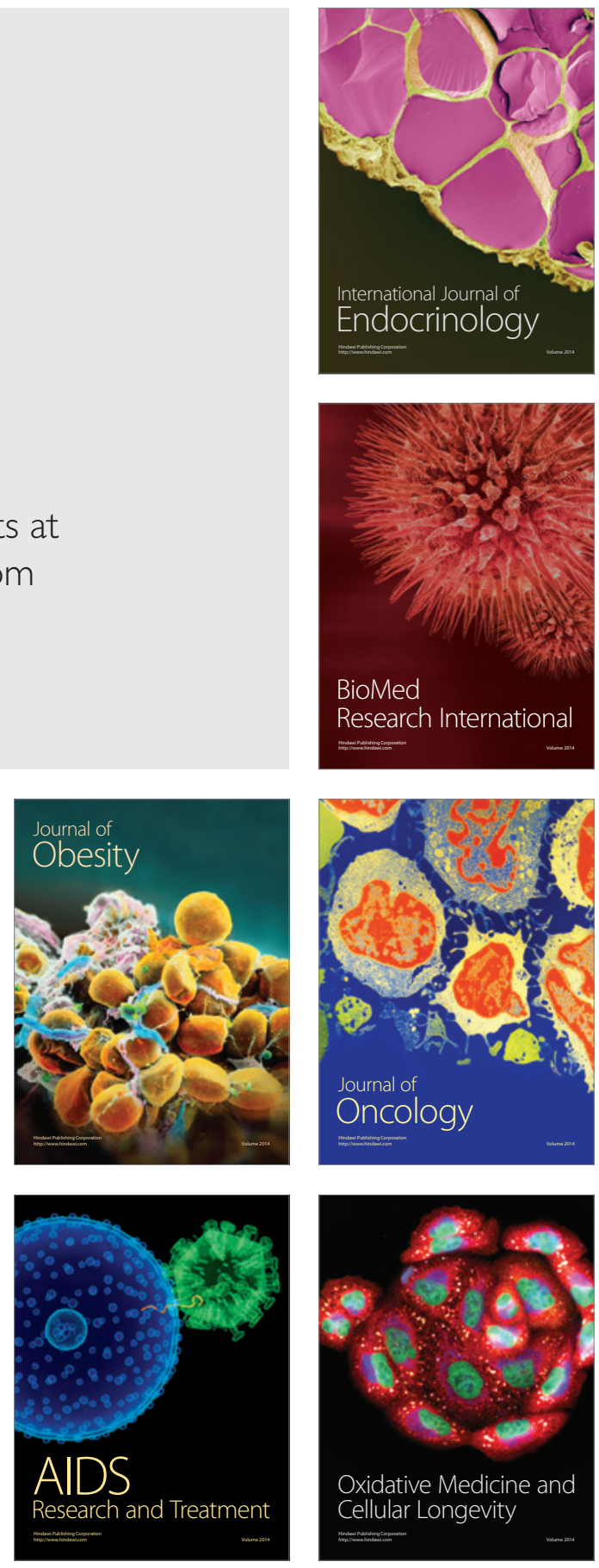